Table I. In vitro effect of hemodilution on whole blood clotting time (seconds)

\begin{tabular}{lllrlr}
\hline & & \multicolumn{3}{c}{ Degree of hemodilution } \\
\cline { 5 - 6 } \multicolumn{1}{c}{ Stimulus } & Pathway & Controls & $25 \%$ & $50 \%$ & $75 \%$ \\
Celite & Intrinsic & $459 \pm 75$ & $442 \pm 67$ & $488 \pm 94$ & $951 \pm 154^{*}$ \\
Thromboplastin & extrinsic & $294 \pm 29$ & $251 \pm 21$ & $277 \pm 34$ & $355 \pm 61$ \\
Thrombin & common & $165 \pm 51$ & $113 \pm 58$ & $91 \pm 34$ & $55 \pm 19$ \\
\hline
\end{tabular}

Values are expressed as mean \pm standard deviation.

*In 9 of 10 samples clotting time was more than 1000 seconds.

Table II. Clinical observation in neonates undergoing cardiac operations

\begin{tabular}{|c|c|c|c|c|c|c|}
\hline & $\begin{array}{c}\text { After } \\
\text { heparinization }\end{array}$ & $\begin{array}{c}5 \mathrm{~min} \\
C P B\end{array}$ & $\begin{array}{c}30 \mathrm{~min} \\
C P B\end{array}$ & $\begin{array}{c}60 \mathrm{~min} \\
C P B\end{array}$ & $\begin{array}{c}90 \mathrm{~min} \\
C P B\end{array}$ & $\begin{array}{l}\text { End } \\
C P B\end{array}$ \\
\hline $\mathrm{ACTc}(\mathrm{sec})$ & $669 \pm 233$ & $980 \pm 63^{*}$ & $992 \pm 63^{*}$ & $995 \pm 15^{*}$ & $1000 \pm 0$ & $774 \pm 214$ \\
\hline HiTT (sec) & $168 \pm 58$ & $163 \pm 80$ & $132 \pm 31$ & $132 \pm 53$ & $118 \pm 53$ & $95 \pm 30$ \\
\hline Heparin (IU/ml) & $2.7 \pm 0.4$ & $1.2 \pm 0.5$ & $1.1 \pm 0.5$ & $1.3 \pm 0.4$ & $1.2 \pm 0.5$ & $0.8 \pm 0.3$ \\
\hline
\end{tabular}

Values are expressed as mean \pm standard deviation. $A C T C$, Celite activated clotting time; HiTT, high-dose thrombin time; CPB, cardiopulmonary bypass. *In 9 of 10 samples ACTc was greater than 1000 seconds.

\section{REFERENCES}

1. Horkay F, Martin P, Rajah SM, Walker DR. Response to heparinization in adults and children undergoing cardiac operations. Ann Thorac Surg 1992;53:822-6.

2. Kriesmer P, Payne NR, Tessmer J, Uden DL. Activated clotting time tests with heparinase in the management of pediatric patients on cardiopulmonary bypass. ASAIO J 1993;39:942-5

3. Gravlee GP, Haddon WS, Rothberger HK, et al. Heparin dosing and monitoring for cardiopulmonary bypass: a comparison of techniques with measurement of subclinical plasma coagulation. J THORAC CARDIOVASC SURG 1990;99:518-27.

4. Culliford AT, Gitel SN, Starr N, et al Lack of correlation between activated clotting time and plasma heparin during cardiopulmonary bypass. Ann Surg 1981;193:105-11.
5. Huyzen RJ, Harder MP, Gallandat Huet RCG, et al. Alternative anticoagulation monitoring during cardiopulmonary bypass in aprotinin treated patients. $\mathbf{J}$ Thorac Cardiovasc Anaesth 1994;8:153-6.

6. Andrew M, Paes B, Milner R, et al. Development of the human coagulation system in the full-term infant. Blood 1987;70:165-72.

7. Kern FH, Morana NJ, Sears JJ, Hickey PR. Coagulation defects in neonates during cardiopulmonary bypass. Ann Thorac Surg 1992;54:541-6.

8. Tabuchi N, de Haan J, Boonstra PW, van Oeveren W. Activation of fibrinolysis in the pericardial cavity during cardiopulmonary bypass. J Thorac CARdiovasc Surg 1993;106:828-33.

\title{
THE USE OF BIOLOGIC GLUE FOR BETTER ADHESIONS BETWEEN THE SKELETAL MUSCLE FLAP AND THE MYOCARDIUM AND FOR INCREASING CAPILLARY INGROWTH
}

\author{
Valeri Chekanov, MD, PhD, Victor Nikolaychik, MD, PhD, and Guennady Tchekanov, MD, Milwaukee, Wis.
}

To date, dynamic cardiomyoplasty relies on use of a properly conditioned, adequately contracting autogenous muscle flap for the correction of myocardial insufficiency. Despite its advantages, cardiomyoplasty has some unresolved problems: (1) poor angiogenic potential of the ischemic, traumatized skeletal muscle and damaged myocardium and (2) incomplete contact between the mobile myocardial wall and the transplanted flap.

From the Milwaukee Heart Project, Milwaukee, Wis.

Received for publication July 19, 1995; accepted for publication Sept. 5, 1995.

J THORaC Cardiovasc Surg 1996;111:678-80

Copyright (C) 1996 by Mosby-Year Book, Inc.

$0022-5223 / 96 \$ 5.00+0 \quad \mathbf{1 2 / 5 4 / 7 1 0 5 9}$
These problems manifest themselves after the opertion. The vascular supply is not reintroduced until 6 to 8 months after the operation. Some patients have died of recurrent myocardial infarction 3 to 5 months after cardiomyoplasty, before a new blood supply from the skeletal muscle to the myocardium had been established. ${ }^{1,2}$ This is the situation that needs to be resolved. Cardiomyoplasty done with skeletal muscle can provide capillary ingrowth to the myocardium, but it may be too late for some patients.

A novel approach to resolving these problems involves "therapeutic angiogenesis." Nine weeks after a modified fibrin glue (containing endothelial cell growth factor) has been applied between the aorta and the left ventricular myocardium, angiographic and histologic investigations have shown newly grown vascular structures between 


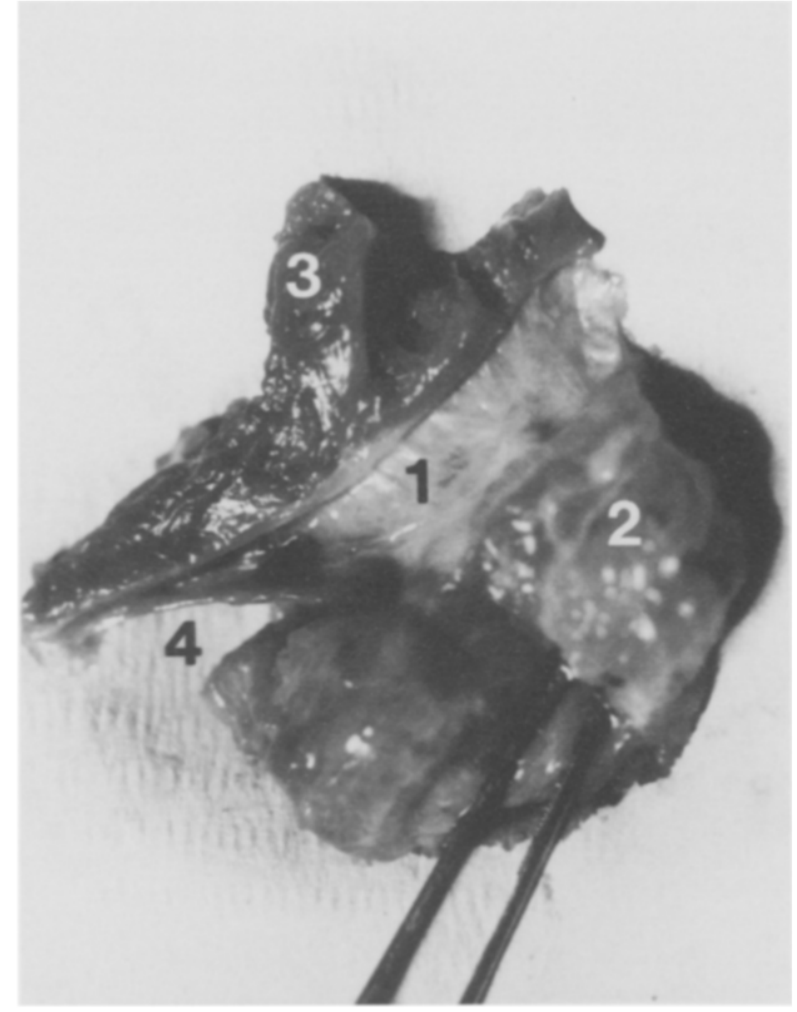

Fig. 1. Macrosection 8 weeks after cardiomyoplasty. Strong adhesions (1) had formed between the latissimus dorsi muscle (2) and the myocardium (3) where the biologic glue was applied. No adhesions had formed in the area without biologic glue (4).

these sites. ${ }^{3}$ The results of this study confirm previous observations that site-directed neovessel formation can be induced by application an angiogenic growth factor. ${ }^{4,5}$

We have implemented the idea of using biologic glue based on a fibrinogen-thrombin composition for cardiomyoplasty. Fibrinogen, the main component of biologic glue, was prepared from donor animals by a standard cryoprecipitation technique. In brief, whole blood obtained from the donor was centrifuged and the plasma decanted. After the plasma had been frozen for 12 hours at $-80^{\circ} \mathrm{C}$, it was thawed at $4^{\circ} \mathrm{C}$ for 4 hours and then centrifuged. The yellowish white precipitate that remained after decantation was collected and stored at $-18^{\circ}$ C. The resulting cryoprecipitate contained concentrated fibrinogen, factor XIII, fibronectin, and vitronectin. In other experiments pure fibrinogen from sheep plasma (F-9754, Sigma Chemical Company, St. Louis, Mo.) was used. Thrombin was purchased from Johnson \& Johnson Patient Care, Inc. (New Brunswick, N.J.). In both cases, final solutions of fibrinogen and thrombin were prepared in separate delivery systems. After mobilization of the latissimus dorsi muscle in adult sheep, the muscle was introduced into the thoracic cavity and wrapped around the heart. The muscle flap was sutured to the myocardium of the left ventricle and two isolated pockets were created.

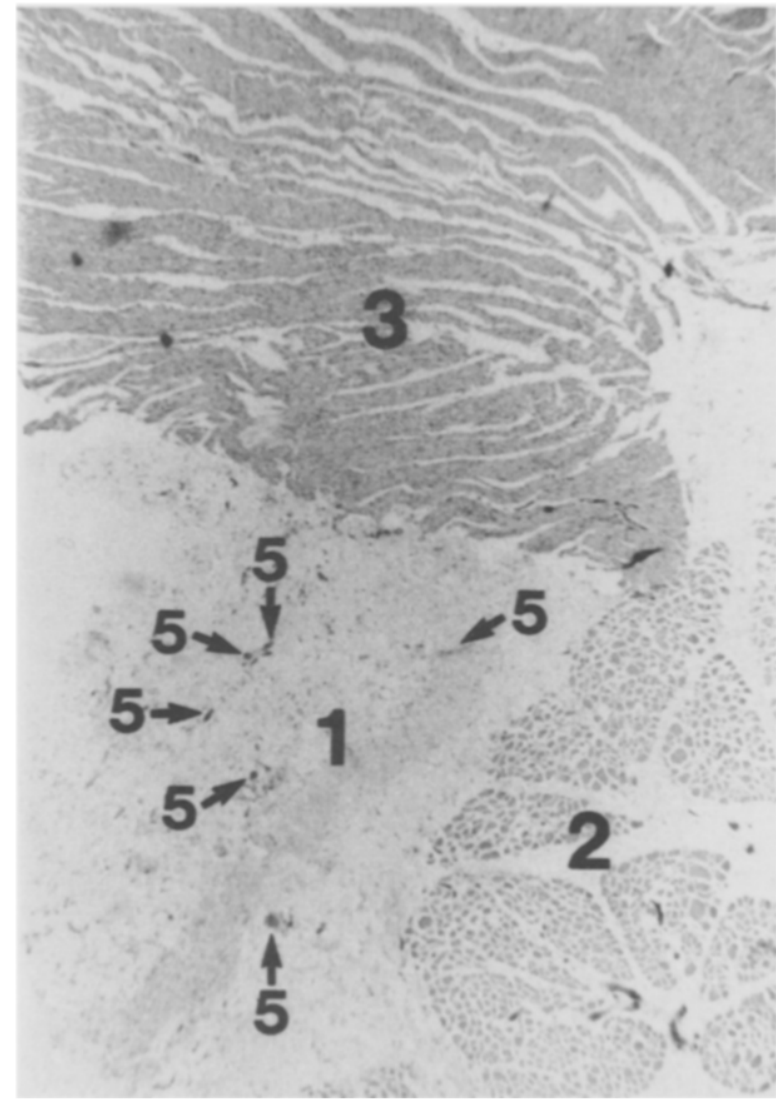

Fig. 2. Histologic examination obtained from a macrosection 8 weeks after cardiomyoplasty and biologic glue application showed vascular growth within the biologic glue (1). Note new blood vessels of significant diameter (5) and growth between the myocardium (3) and the latissimus dorsi muscle (2).

In one of the pockets the biologic glue was introduced by simultaneously applying the two separate solutions of fibrinogen and thrombin between the skeletal muscle and myocardium. The amount of glue used per animal was in the range of 20 to $30 \mathrm{ml}$. As a control, no biologic glue was introduced in the other pocket. After the operation the animals had the usual postoperative management and the typical stimulation protocol for skeletal muscle training.

After 8 weeks we examined the muscle flap and the myocardium of the left ventricle (Fig. 1). We saw extremely strong adhesions between the latissimus dorsi muscle and the myocardium in the pocket where the biologic glue had been applied. Histologic examination showed new blood vessel growth of significant diameter (Fig. 2). No adhesions had developed in the areas in which biologic glue was not used.

In summary, we have shown that we can induce significant formation of new blood vessel structures and produce strong adhesions between the myocardium and skeletal muscle with the use of biologic glue. This finding may be helpful for patients undergoing cardiomyoplasty. 
Enhancement of angiogenesis and vasculogenesis after cardiomyoplasty might be achieved by local implantation of autologous endothelial cells cultured ex vivo or by application of angiogenic factors like vascular endothelial growth factor, platelet-derived growth factor, insulin-like growth factor, fibroblast growth factor, or extracellular matrix components like fibronectin, laminin, and proteoglycans. From this point of view, the fibrin meshwork serves only as a local delivery depot. However, it seems to us that a pure fibrin meshwork can sufficiently mimic the extracellular matrix for endothelial cells and support their growth. We believe that further study is required to assess the potential of various growth factors and suppressors of ischemia- reperfusion lesions in shortening the recovery period between surgery and full assistance in pumping functions of the native heart.

\section{REFERENCES}

1. Carpentier A, Chachques JC, Acar C, et al. Dynamic cardiomyoplasty at seven years. J ThORAC CARDIOvasC SuRg 1993; 106:42-54.

2. Chekanov V, Krakovsky A, Buslenko N, et al. Cardiomyoplasty: review of early and late results. Vasc Surg 1994;28:481-8.

3. Fasol R, Schumacher B, Schlaudraff K, et al. Experimental use of a modified fibrin glue to induce site-directed angiogenesis from the aorta to the heart. J THORAC CARdiovasc SuRG 1994;107:1432- 9 .

4. Janagisawa-Miwa A, Uchida Y, Nakamurea F, et al. Salvage of infarcted myocardium by angiogenic action of basic fibroblast growth factor. Science 1992;257:1401-2.

5. Banai S, Jaklitsch M, Casscells W, et al. Effect of acidic fibroblast growth factor on normal and ischemic myocardium. Circ Res 1991;69:76-85. 